\title{
Consideraciones nutricionales en entrenamiento en altura
}

\author{
Nutritional considerations in altitude training \\ Considerações nutricionais no treino em altitude
}

\author{
Mauricio Serrato Roa ${ }^{{ }^{*}}$
}

Recibido: 1 de julio de 2019. Aceptado para publicación: 5 de agosto de 2019 https://doi.org/10.35454/rncm.v2n2.010

\begin{abstract}
Resumen
La altitud, moderada y alta, somete al individuo a un ambiente hostil al cual debe adaptarse. La hipoxia hipobárica causa una reducción en la presión arterial de oxígeno que produce una disminución significativa en la capacidad de generar energía, reduciendo el consumo máximo de oxígeno. Se desencadenan respuestas agudas a medida que las respuestas adaptativas se instauran. Para lograr adaptarse a la altura es necesario implementar medidas nutricionales específicas que serán definitivas para favorecer este proceso. Se deben instaurar medidas para evitar el catabolismo, lograr la adecuación nutricional, el estado de hidratación, la protección ante el estrés oxidativo, así como favorecer el aumento de la capacidad de transporte de oxígeno. Estas medidas deben hacerse en forma periódica según el plan de entrenamiento, el tipo de deporte y otras variables individuales.
\end{abstract}

Palabras clave: altura, hipoxia hipobárica, nutrición, deporte.

\begin{abstract}
Moderate and high altitude subject the individual to a hostile environment, for which one has to adapt. Hypobaric hypoxia causes a reduction in blood pressure of oxygen, which causes a significant decrease in the ability to produce energy, reducing the maximum consumption of oxygen. Acute responses are triggered, while adaptive responses are instituted. In order to adapt to altitude, it is necessary to implement specific nutritional measures that will be definitive in order to favor this process. Measures must be put in place to prevent catabolism, to reach nutritional adequacy, hydration status, for protection against oxidative stress, as well as to favor an increase in oxygen transport capacity. These measures should be periodized according to the training plan, the type of sport and other individual variables.
\end{abstract}

Key words: Altitude; Hypobaric hypoxia; Nutrition; Sport.

\section{Resumo}

A altitude, moderada e alta, sujeita o indivíduo a um ambiente hostil ao qual ele deve se adaptar. A hipoxia hipobárica provoca uma redução na pressão arterial de oxigênio que produz uma diminuição significativa na capacidade de gerar energia, reduzindo o consumo máximo de oxigênio. As respostas agudas são desencadeadas à medida que as respostas adaptativas são estabelecidas. Para adaptar-se à altura é necessário implementar medidas nutricionais específicas que serão definitivas para promover esse processo. Devem ser introduzidas medidas para evitar o catabolismo, alcançar a adequação nutricional, o estado de hidratação, a proteção contra o estresse oxidativo, bem como favorecer uma maior capacidade de transporte de oxigénio. Essas medidas devem ser realizadas periodicamente de acordo com o plano de treino, o tipo de desporto e outras variáveis individuais.

Palavras-chave: altitude, hipoxia hipobárica, nutrição, desporto.

\section{INTRODUCCIÓN}

Durante las migraciones las especies han desarrollado la habilidad de soportar el paso por las altas montañas.

1 Posgrado Medicina del Deporte. Universidad Nacional de Colombia, Bogotá, D.C.

Ministerio del Deporte, Colombia.
Muchas de ellas se establecieron en asentamientos en planicies elevadas logrando desarrollar evolutivamente mecanismos compensatorios a la hipoxia hipobárica ${ }^{(1)}$. 
La exposición a alturas moderadas o altas $(2.000$ a $5.600 \mathrm{msnm}$ ) causa una disminución de la presión barométrica que reduce el gradiente de presión para que ocurra la difusión del oxígeno desde el ambiente hasta la mitocondria. En este proceso la caída de la presión alveolar de oxígeno $\left(\mathrm{PAO}_{2}\right)$ causa a su vez una reducción de la presión arterial de $\mathrm{O}_{2}\left(\mathrm{PaO}_{2}\right)$, hecho conocido como hipoxia. $\mathrm{La} \mathrm{PaO}_{2}$ reducida causa una disminución en la saturación de la hemoglobina $\left(\mathrm{SaO}_{2}\right)$, en especial cuando la $\mathrm{PaO}_{2}$ cae por debajo de $60 \mathrm{mmHg}$ debido a que, en esta zona de la curva de disociación de la $\mathrm{Hb}$, pequeños cambios en la presión afectan grandemente la $\mathrm{SaO}_{2}$. La desaturación causa reducción del aporte de $\mathrm{O}_{2}$ a los tejidos ya que del total de $\mathrm{O}_{2}$ que la hemoglobina $(\mathrm{Hb})$ puede transportar solo una fracción estará unida al $\mathrm{O}_{2}$. Un menor volumen de $\mathrm{O}_{2}$ llega a los tejidos causando una serie de alteraciones tisulares que resultan en cambios fisiológicos, bioquímicos, psicológicos y metabólicos entre otros, que afectan la función global de individuo, así como el rendimiento físico.

Estos cambios agudos provocan respuestas compensatorias. La hipoxia induce una respuesta en los quimiorreceptores que a su vez estimulan el centro respiratorio en el tallo cerebral, haciendo que aumente la ventilación (VE) pulmonar para incrementar secundariamente la $\mathrm{PAO}_{2}$.

Mientras las respuestas agudas funcionan de manera temporal, se ponen en marcha respuestas adaptativas más eficientes. Los quimiorreceptores en el aparato yuxtaglomerular del riñón aumentan la producción de eritropoyetina (EPO) con el fin de aumentar la producción medular de glóbulos rojos y su concentración de hemoglobina. Otros mecanismos parecen estar involucrados en las adaptaciones crónicas diferentes a los cambios hematológicos. Como resultado se produce una elevación sostenida de la masa total de hemoglobina $\left(\mathrm{Hbt}_{\text {mass }}\right)(1)$. Un individuo a nivel del mar puede tener una $\mathrm{HBt}_{\text {mass }}$ de $8 \mathrm{~g} / \mathrm{kg}$ mientras que un individuo de la altura puede llegar a tener $12 \mathrm{~g} / \mathrm{kg}$ y si entrena en altura este valor puede ser $15 \mathrm{~g} / \mathrm{kg}$. Con el tiempo se logran otras adaptaciones musculares, ventilatorias y cardiovasculares ${ }^{(2)}$.

Todos los cambios agudos y crónicos con la altura causan cambios muy importantes en el comportamiento alimentario. De hecho, se reduce el apetito mientras se incrementan las necesidades de hierro, proteínas, carbohidratos y líquidos. En este sentido se debe ajustar el aporte nutricional y las características de los alimentos para los individuos que van a permanecer en altura, con medidas nutricionales muy específicas que permitan lograr una buena adaptación y ayudar a com- pensar tanto la pérdida del rendimiento físico como la reducción del tejido magro.

Muchos deportistas de nivel del mar cuando compiten en altura deben buscar el menor impacto en su rendimiento mediante estrategias de diversa índole. Otros buscan adaptarse a la altura para mejorar su rendimiento a nivel del mar, ya sea viviendo arriba y entrenando abajo (LHTL), viviendo arriba y entrenando arriba (LHTH) o mediante protocolos de entrenamiento con hipoxia intermitente (HIT), razones por las cuales se requieren estrategias diferentes para cada situación.

Las demandas energéticas cambian en la exposición a la altura, el aumento de la tasa metabólica puede afectar la composición corporal. En estas condiciones los individuos se encuentran expuestos a un mayor estrés oxidativo, por lo que se requiere suplementación de antioxidantes junto con una dieta rica en los mismos. La dieta debe ser modificada para intentar reducir la producción de gas intestinal.

Resulta fundamental realizar un buen plan nutricional para personas y atletas que van a competir o entrenar en altura de manera que se pueda ayudar a lograr el resultado esperado en este ambiente de por sí difícil. Esta revisión se centrará en los cambios que ocasionan la disminución del apetito, la deshidratación provocada por la altura y los requerimientos aumentados de hierro y antioxidantes, especialmente en mujeres que se exponen a alturas intermedias. Es necesario tener en cuenta que la mayoría de los estudios publicados se refieren a grandes altitudes y no a las alturas moderadas que son las que conciernen a esta revisión.

\section{ANÁLISIS Y DISCUSIÓN}

Los individuos que practican deportes en los cuales se involucra de alguna manera la resistencia aeróbica, verán afectado de manera significativa su rendimiento en la altura. La hipoxia hipobárica (menor presión atmosférica por lo tanto menor gradiente para la difusión del $\mathrm{O}_{2}$ ) causa una reducción en la disponibilidad mitocondrial de $\mathrm{O}_{2}$, motivo por lo cual se reduce su extracción periférica, hecho que conlleva a una reducción en producción de energía aeróbica. Según Fulco ${ }^{(3)}$ por cada 100 metros por encima de los $1.500 \mathrm{msnm}$ el consumo máximo de oxígeno $\left(\dot{\mathrm{V}} \mathrm{O}_{2 \max }\right)$ se reduce en $1 \%$ por cada 100 adicionales de altitud. Fisiológicamente por cada $1 \%$ de disminución en la $\mathrm{SaO}_{2}$ por debajo de $95 \%$ el $\dot{\mathrm{V}}{ }_{2 \text { max }}$ se reduce en $1 \%-2 \%{ }^{(4)}$.

Los efectos secundarios de la hipoxia derivan en primera instancia de la deficiencia relativa de oxígeno 
para el trabajo muscular y en segundo plano como efectos secundarios de las respuestas agudas al estrés que provoca dicha hipoxia. El aumento inmediato y permanente en la VE en altura, causa también un incremento en la pérdida insensible de agua por la respiración ${ }^{(5)}$. Durante estos primeros días se siente fatiga, cefalea y disminución del apetito ${ }^{(6)}$.

La exposición crónica a la altura provoca un aumento de la $\mathrm{HB}_{\text {tmass }}$ como resultado del aumento de la producción de $\mathrm{EPO}^{(3)}$. Se ha reportado que hay una reducción en el costo energético del ejercicio a nivel del mar debido a un metabolismo más eficiente al producir más ATP por mol de $\mathrm{O}_{2}$, junto con un menor consumo de ATP ${ }^{(6)}$. Los principales mediadores de los efectos celulares de la altura se deben al aumento tanto del Factor Inducible de Hipoxia (HIF-1) como del factor de crecimiento vascular endotelial (VEGF). El efecto de este factor causa aumento de ciertas proteínas críticas en el metabolismo energético, como la enzima piruvato deshidrogenasa (PDH), lactato deshidrogenasa (LDH), el transportador de glucosa GLUT-1 y el transportador de monocarboxilados MCT-4. Todos en conjunto causan un aumento de la glicolisis citoplasmática que lógicamente termina aumentando la producción de lactato en cargas submáximas. Con la aclimatación se reduce la producción de lactato en condiciones hipóxicas, efecto que se ha denominado "La paradoja del Lactato"(7).

Resulta evidente que el rendimiento de resistencia se afectará proporcionalmente con el nivel de hipoxia al cual el individuo será sometido. Para favorecer las adaptaciones a la altura, es necesario complementar las medidas fisiológicas con medidas nutricionales con el fin de evitar la deshidratación, reducción de la masa magra, el estrés oxidativo y aportar macronutrientes para aumentar la síntesis proteica, de hemoglobina y glóbulos rojos.

\section{MEDIDAS NUTRICIONALES PARA INCREMENTAR LOS DEPÓSITOS DE HIERRO EN ALTURA}

Una de las medidas más importantes para favorecer las adaptaciones en la altura es mejorar la capacidad de conducción y utilización del $\mathrm{O}_{2}$ aumentando la cantidad de hemoglobina. La síntesis de esta proteína es dependiente de la presencia del hierro tisular disponible ${ }^{(8)}$.

El incremento en la eritropoyesis en la altura causa un flujo del hierro de los depósitos tisulares haciendo que las reservas se depleten parcialmente. $\mathrm{Al}$ inicio se puede ver una reducción en los niveles de ferritina ${ }^{(9)}$.
En condiciones normales una dieta rica en hierro de alto valor biológico puede compensar las necesidades aumentadas en la altura; sin embargo, en el caso de las mujeres en edad reproductiva, es probable que requieran suplementación oral desde unas semanas antes de la exposición a la altura. Se recomienda realizar un monitoreo frecuente de la ferritina, y en caso de que se reduzca, suplementar $100 \mathrm{mg} /$ día $^{(10)}$.

\section{MEDIDAS NUTRICIONALES PARA MANTENER EL BALANCE ENERGÉTICO}

Durante el periodo de aclimatación a la altura, el apetito se ve francamente disminuido ${ }^{(11)}$, hecho que junto a un incremento en la tasa metabólica basal (TMB) ${ }^{(12)}$, causan un balance energético negativo. Este efecto en la reducción del apetito es más significativo en alturas superiores a $5.000 \mathrm{msnm}$. El catabolismo proteico puede estar relacionado con la activación de la $\mathrm{AMPK}^{(13)}$. Un ajuste apropiado del gasto energético junto con la ingesta de nutrientes puede ayudar a prevenir la reducción de la masa magra ${ }^{(14)}$. La reducción en el peso también obedece en parte a la deshidratación causada por las pérdidas insensibles de agua debido al aumento compensatorio de la VE y de la diuresis ${ }^{(15)}$. Es claro que una adecuada hidratación es clave para el rendimiento deportivo. El aumento de la temperatura central producto de hipermetabolismo del ejercicio implica que se desencadenen los mecanismos compensatorios de termorregulación. Para favorecer la pérdida de calor es necesario realizar una desviación del gasto cardiaco, que estaba destinado al músculo, hacia la piel para luego secretar sudor y disipar la energía térmica por evaporación. Las pérdidas obligatorias de agua se ven incrementadas en la altura, tanto que pueden llegar en promedio hasta $2.700 \mathrm{~mL}$ en un hombre. Si estas pérdidas se suman al volumen de sudoración por ejercicio, el individuo estará más predispuesto a sufrir grados importantes de deshidratación. El uso de bebidas energéticas, con dosis pequeñas de cafeína, no altera el balance hídrico durante el ejercicio ${ }^{(16)}$, pero sí mantiene su efecto ergogénico en dosis que pueden ir hasta los $8 \mathrm{mg} / \mathrm{kg}$.

Se debe aumentar la ingesta de carbohidratos y proteínas en altura para poder mantener el peso corporal y reponer los depósitos de carbohidratos. Un aumento de la TMB afecta $65 \%$ del total de gasto energético diario, por lo tanto, la ingesta calórica debe aumentar en forma proporcional. Se recomienda que el atleta aumente la frecuencia de alimentación, haciendo énfasis en un contenido de carbohidratos que proporcionen como mínimo $60 \%$ de las necesidades energéticas diarias. 
Los carbohidratos son además un buen substrato en altura ya que para oxidarse, consumen menos oxígeno que las grasas. Los deportes de resistencia pueden requerir dietas ricas en carbohidratos incluso hasta 80 $\%$ del gasto calórico diario, lo que da un valor cercano a los $13 \mathrm{~g} / \mathrm{kg}$. En el deporte se hace énfasis en los carbohidratos líquidos como suplementos ergogénicos, los cuales están disponibles comercialmente como maltodextrinas en diferentes combinaciones en barras energéticas, gomas o geles. Hoy en día muchos se asocian a dosis de cafeína que van desde 25 hasta $175 \mathrm{mg}$. Dosis que son consideradas muy bajas para afectar el balance hídrico ${ }^{(16)}$. La cafeína, en su forma anhidra, aumenta la atención durante ejercicios prolongados, mejora la resistencia y el tiempo de fatiga, entre otros ${ }^{(16)}$.

En la recuperación postejercicio se recomienda el uso de carbohidratos mixtos en combinación con proteínas. Estas bebidas están disponibles en concentraciones variables, con contenido alto de leucina y carbohidratos de rápida absorción. Se ha demostrado que esta combinación ayuda a mejorar la resíntesis de glucógeno $^{(17)}$. Para esta recuperación se deben consumir $15 \mathrm{~g} / \mathrm{kg} / \mathrm{h}$ de carbohidratos en las primeras 4 horas postejercicio $^{(18)}$.

En la actualidad se está estudiando la adición de curcumina y bromelina a las bebidas de recuperación por su efecto en la reducción de la inflamación postejercicio $^{(19)}$. De acuerdo con Jeukendrup, las necesidades nutricionales deben ser personalizadas y ajustadas en cada paciente y periodizadas de acuerdo con el plan de entrenamiento ${ }^{(20)}$.

Adicionar vitamina $\mathrm{D}$ podría ser útil en condiciones de hipoxia hipobárica para prevenir las pérdidas de masa magra ${ }^{(21)}$, además de que ayuda a reducir la hidroxilación de la vitamina $\mathrm{D}_{3}$ en el riñón como consecuencia de la hipoxia.

\section{MEDIDAS NUTRICIONALES PARA EVITAR EL ESTRÉS OXIDATIVO EN ALTURA}

Se ha establecido que el ejercicio de resistencia conlleva un estrés oxidativo adicional debido al alto flujo mitocondrial de electrones que causa un aumento de las Especies Reactivas de Oxígeno o Nitrógeno (RONS, por sus siglas en inglés). Estos radicales reactivos deben ser barridos por los sistemas celulares de antioxidantes, que por fortuna son inducidos precisamente por entrenamiento como medida compensatoria y adaptativa a la resistencia. El estrés oxidativo ha sido asociado al inicio del Síndrome de Dolor Tardío Postejercicio (DOMS, por sus siglas en inglés) y el excesivo entrenamiento ${ }^{(22)}$.

Aún es contradictoria la información sobre los efectos de la suplementación de antioxidantes en el ejercicio de resistencia, sin embargo al considerar que la exposición a la altura puede incrementar la producción de RONS en las células musculares por el ejercicio, se ha sugerido que la suplementación de antioxidantes puede prevenir el daño celular, ayudando como segunda línea de defensa a los sistemas celulares como la superóxido dismutasa (SOD), glutatión peroxidasa GPX) catalasa (CAT) o glutatión reductasa (GR).

Las vitaminas $\mathrm{E}$ y A en la membrana celular y la vitamina $C$ intracelular ayudan como segunda línea de defensa ante los RONS. Algunos autores han reportado algún beneficio de su uso en ejercicio en altura ${ }^{(23)}$. El uso de la vitamina $E$ se ha recomendado con este $\mathrm{fin}^{(23)}$; sin embargo, se debe esperar aún más evidencia que soporte esta estrategia para poder hacer una recomendación ${ }^{(24)}$. El uso de la mega dosis podría incluso obstruir el proceso de señalización para lograr la adaptación al entrenamiento ${ }^{(25)}$.

La recomendación más clara hace referencia al consumo de alimentos ricos en antioxidantes naturales contenidos en frutas y vegetales con los cuales se puede minimizar el estrés oxidativo (vitamina $\mathrm{C}$, carotenos, polifenoles, resveratrol, glutatión, quercetina, flavonoides y otros fitoquímicos) $)^{(24,26)}$.

\section{RECOMENDACIONES}

Resulta necesaria una adecuada planificación nutricional para los atletas que se van a exponer a alturas moderadas y altas, debido a los efectos secundarios de la altura en rendimiento y potencialmente en la salud. Por lo tanto, se deben implementar medidas especiales para mantener un adecuado estado de hidratación, evitar la pérdida de masa magra, proteger del daño oxidativo y favorecer las adaptaciones hematológicas y musculares.

Los requerimientos aumentados de algunos macro y micronutrientes deben ser ajustados de manera individual teniendo en cuenta la altura a la cual se exponen, el peso, sexo y modalidad deportiva. Estas recomendaciones están basadas en los requerimientos de los deportes con un componente de resistencia importante.

Se debe diferenciar si el deportista se expondrá a la altura buscando adaptarse para obtener beneficios, o simplemente debe competir en altura como consecuencia de su calendario competitivo. Por lo tanto, los 
ajustes son diferentes y la nutrición debe ser adecuada de manera específica, ya que en la primera situación se busca permanecer mínimo 2 a 3 semanas para lograr adaptaciones, mientras que en la segunda se debe tratar de atenuar el impacto de altura en el rendimiento y los síntomas agudos de la exposición a la altura.

En caso de la exposición aguda para competir en altura se busca que los deportistas ingieran cantidades suficientes de carbohidratos para carga de glucógeno, suplementarlos como carbohidratos líquidos, evitar alimentos que aumenten el gas intestinal, incrementar el consumo de alimentos con antioxidantes naturales y suplementar las vitaminas E y C. También se recomienda la hidratación para mantener el balance hídrico, monitorizando el peso y la densidad urinaria continuamente. Durante la competencia se puede usar la cafeína sin el riesgo de aumentar la deshidratación.

\section{CONCLUSIÓN}

Realizar un plan nutricional para el deportista que se va a exponer a alturas moderadas es fundamental para lograr el efecto adaptativo y de rendimiento esperados. Para ello se debe realizar una periodización nutricional individual con el fin de evitar el catabolismo, la deshidratación, la pérdida de hierro tisular y el estrés oxidativo. Esta intervención debe ser integral y realizada por el equipo multidisciplinario.

\section{Agradecimientos}

Agradecimientos a la doctora Johana Ledezma nutricionista por la revisión de este documento.

\section{Declaración de autoría}

Este escrito es autoría de revisión de la literatura y experiencias propias del autor.

\section{Declaración de conflictos de interés y financiamiento}

No refiero conflicto de intereses y no recibo aporte de la industria de productos nutricionales o farmacéuticos relacionados con este escrito.

\section{Referencias bibliográficas}

1. Böning D. Physical Exercise at Altitude - Acclimation and Adaptation Effects in Highlanders on Different Continents. Dtsch Z Sportmed. 2019;70:135-40.
2. van der Zwaard S, Brocherie F, Kom BLG, Millet GP, Deldicque $\mathrm{L}$, van der Laarse WJ, et al. Adaptations in muscle oxidative capacity, fiber size, and oxygen supply capacity after repeatedsprint training in hypoxia combined with chronic hypoxic exposure. J Appl Physiol (1985). 2018;124(6):1403-12.

3. Fulco CS, Rock PB, Cymerman A. Maximal and submaximal exercise performance at altitude. Aviat Space Environ Med. 1998;69(8):793-801.

4. Dempsey JA, Wagner PD. Exercise-induced arterial hypoxemia. J Appl Physiol (1985). 1999;87(6):1997-06.

5. Schmidt W, Heinicke K, Rojas J, Manuel Gomez J, Serrato M, Mora M, et al. Blood volume and hemoglobin mass in endurance athletes from moderate altitude. Med Sci Sports Exerc. 2002;34(12):1934-40.

6. Czuba M, Maszczyk A, Gerasimuk D, Roczniok R, FidosCzuba O, Zając A, et al. The effects of hypobaric hypoxia on erythropoiesis, maximal oxygen uptake and energy cost of exercise in normoxia in elite biathletes. J Sports Sci Med. 2014; 13(4):912-20.

7. Hochachka PW, Beatty Cl, Burelle Y, Trump ME, McKenzie DC, Matheson GO. The lactate paradox in human highaltitude physiological performance. News Physiol Sci. 2002;17:122-6.

8. Pollycove M, Mortimer R. The quantitative determination of iron kinetics and hemoglobin synthesis in human subjects. J Clin Invest. 1961;40:753-82.

9. Pauls DW, van Duijnhoven H, Stray-Gundersen J. Iron insufficient erythropoiesis at altitude-speed skating. Med Sci Sports Exerc. 2002;34(5):252S.

10. Nandadeva TDP, Dissanayake AMSDM, Rajaratne AAJ, Nanayakkara SDI. Effect of iron supplementation during high altitude training on haemoglobin and iron status of Sri Lankan middle-and long-distance athletes. Sri Lanka Journal of Medicine. 2019;28(1):30-40.

11. Kayser B. Nutrition and energetics of exercise at altitude. Theory and possible practical implications. Sports Med. 1994;17(5):309-23.

12. Butterfield GE, Gates J, Fleming S, Brooks GA, Sutton JR, Reeves JT. Increased energy intake minimizes weight loss in men at high altitude. J Appl Physiol (1985). 1992;72(5):1741-8.

13. Liu L, Cash TP, Jones RG, Keith B, Thompson CB, Simon MC. Hypoxia-induced energy stress regulates mRNA translation and cell growth. Mol Cell. 2006; 21(4):521-31.

14. Dünnwald $T$, Gatterer $H$, Faulhaber $M$, Arvandi $M$, Schobersberger W. Body Composition and Body Weight Changes at Different Altitude Levels: A Systematic Review and Meta-Analysis. Front physiol. 2019; 10:430.

15. Richardson A, Watt P, Maxwell N. Hydration and the physiological responses to acute normobaric hypoxia. Wilderness Environ Med. 2009;20(3):212-20.

16. Goldstein ER, Ziegenfuss T, Kalman D, Kreider R, Campbell $\mathrm{B}$, Wilborn $\mathrm{C}$, et al. International society of sports nutrition 
position stand: caffeine and performance. J Int Soc Sports Nutr. 2010;7(1):5.

17. Beelen M, Burke LM, Gibala MJ, van Loon LJC. Nutritional strategies to promote postexercise recovery. Int J Sport Nutr Exerc Metab. 2010; 20(6):515-32.

18. Burke LM, Hawley JA, Wong SH, Jeukendrup AE. Carbohydrates for training and competition. J Sports Sci. 2011; 29 Suppl 1: S17.

19. Heaton LE, Davis JK, Rawson ES, Nuccio RP, Witard OC, Stein $\mathrm{Kw}$, et al. Selected in-season nutritional strategies to enhance recovery for team sport athletes: a practical overview. Sports Me. 2017; 47(11): 2201-8.

20. Jeukendrup A. A step towards personalized sports nutrition: Carbohydrate intake during exercise. Sports Med. 2014;44(Suppl 1):25-33.

21. Kasprzak Z, Sliwocka E, Henning K, Pilaczyńska-Szczesniak Ł, Huta-Osiecka A, Nowak A. Vitamin D, Iron metabolism, and diet in alpinists during a 2-week high-altitude climb. High Alt Med Biol. 2015;16(3):230-5.
22. Kawamura T, Muraoka I. Exercise-induced oxidative stress and the effects of antioxidant intake from a physiological viewpoint. Antioxidants (Basel). 2018;7(9): pii: E119.

23. Bryant RJ, Ryder J, Martino P, Kim J, Craig BW. Effects of vitamin $\mathrm{E}$ and $\mathrm{C}$ supplementation either alone or in combination on exercise-induced lipid peroxidation in trained cyclists. J Strength Cond Res. 2003;17(4):792-800.

24. Koivisto AE, Olsen T, Paur I, Paulsen G, Bastani NE, Garthe I, et al. Effects of antioxidant-rich foods on altitude-induced oxidative stress and inflammation in elite endurance athletes: A randomized controlled trial. PloS One. 2019;14(6): e0217895.

25. Ristow M, Zarse K, Oberbach A, Klöting N, Birringer M, Kiehntopf M, et al. Antioxidants prevent health-promoting effects of physical exercise in humans. Proc Natl Acad Sci USA. 2009; 106(21):8665-70.

26. Kayser B. Nutrition and high altitude exposure. Int J Sports Med. 1992;13 (Suppl 1):S129-32. 